\title{
Alternative Scenarios of Relativistic Heavy-Ion Collisions: I. Baryon Stopping
}

\author{
Yu.B. Ivanov, ${ }^{1, *}$ \\ ${ }^{1}$ Kurchatov Institute, Moscow RU-123182, Russia
}

\begin{abstract}
Simulations of relativistic heavy-ion collisions within the three-fluid model employing a purely hadronic equation of state (EoS) and two versions of the EoS involving deconfinement transition are presented. The latter are an EoS with the first-order phase transition and that with a smooth crossover transition. The model setup is described in detail. The analysis is performed in a wide range of incident energies $2.7 \mathrm{GeV} \leq \sqrt{s_{N N}} \leq 39 \mathrm{GeV}$ in terms of the center-of-mass energy. Results on proton and net-proton rapidity distributions are reported. Comparison with available data indicate certain preference of the crossover EoS. It is found that predictions within deconfinementtransition scenarios exhibit a "peak-dip-peak-dip" irregularity in the incident energy dependence of the form of the net-proton rapidity distributions in central collisions. This irregularity is a signal of deconfinement onset occurring in the hot and dense stage of the nuclear collision.
\end{abstract}

PACS numbers: 25.75.-q, 25.75.Nq, 24.10.Nz

Keywords: relativistic heavy-ion collisions, baryon stopping, hydrodynamics, deconfinement

\section{INTRODUCTION}

Over the last decade relativistic heavy ion physics has made tremendous progress in understanding of the QCD phase diagram in the domain of high temperatures and low baryon density [1]. However, a number of important questions still remain open. These are: At which incident energy an onset of deconfinement happen? What is the order of the deconfinement transition at high baryon densities? Is there a critical end point in the phase diagram? These questions form the main motivation for the currently running beam-energy-scan program [2] at the Relativistic Heavy-Ion Collider (RHIC) at Brookhaven National Laboratory (BNL) and low-energy-scan program [3] at Super Proton Synchrotron (SPS) of the European Organization for Nuclear Research (CERN), as well as newly constructed Facility for Antiproton and Ion Research (FAIR) in Darmstadt [4] and the Nuclotron-based Ion Collider Facility (NICA) in Dubna [5].

This paper starts a series of papers, in which I hope to shed light on two first questions formulated above. In these papers I will report results of thorough simulations of relativistic heavy-ion collisions in the energy range from $2.7 \mathrm{GeV}$ to $39 \mathrm{GeV}$ (in terms of centerof-mass energy, $\sqrt{s_{N N}}$ ). This domain covers the energy range of the RHIC beam-energy-scan and SPS lowenergy-scan programs, as well as energies of the future FAIR and NICA facilities and the Alternating Gradient Synchrotron (AGS) at BNL. Though experiments at the AGS have been already stopped, experimental data taken at the AGS are still unique since they were neither updated nor repeated in any newer measurements so far. The simulations were performed within a model of the three-fluid dynamics (3FD) [6] employing three different equations of state (EoS): a purely hadronic EoS 7] (hadr. EoS), which was used in the major part of the 3FD simu-

*e-mail: Y.Ivanov@gsi.de lations so far [6, 8 11], and two versions of EoS involving the deconfinement transition [12]. These two versions are an EoS with the first-order phase transition and that with a smooth crossover transition. Since neither of EoS's includes a critical end point, these simulations do not touch the last question formulated above.

This report is planned as a series of papers because it concerns a great number of bulk observables (rapidity and transverse spectra, flow observables and multiplicities) for various species and a large number of incident energies, their comparison with available data, and also illustrations of global evolution of collisions within different scenarios (i.e. EoS's). Analysis of whole the set of observables will be useful for revealing possible correlations in the energy evolution of these observables within different scenarios, which can be used as experimental indications of the deconfinement onset or its absence.

It is reasonable to start this series with analysis of the baryon stopping because a degree of stopping of colliding nuclei is one of the basic characteristics of the collision dynamics, which determines a part of the incident energy of colliding nuclei deposited into produced fireball and hence into production of secondary particles. The deposited energy in its turn determines the nature (hadronic or quark-gluonic) of the produced fireball and thereby its subsequent evolution. Therefore, a proper reproduction of the baryon stopping is of prime importance for theoretical understanding of the dynamics of the nuclear collisions. I will argue that certain irregularity in the incident-energy dependence of the baryon stopping may indicate an onset of deconfinement.

The paper is organized as follows. In the next section III a brief survey of the 3FD model is presented: basic ideas and choice of parameters relevant for the present simulations. Properties of the EoS's used in the present simulations are illustrated in sect. III] Predictions of proton and net-proton rapidity distributions and their comparison with available experimental data are presented in sect. [V] Analysis of the form of these rapidity distributions and its evolution with incident energy rise is 
done in sect. V] In sect. VI a summury of results is formulated. Results on baryon stopping in simulations with deconfinement transitions have been already briefly reported (without details) in Ref. [13].

\section{3FD MODEL}

The 3FD model [6] is a straightforward extension of the 2-fluid model with radiation of direct pions 14 16 and (2+1)-fluid model [17, 18]. The above models were extend in such a way that the created baryon-free fluid (which is called a "fireball" fluid, following the Frankfurt group) is treated on equal footing with the baryon-rich ones. A certain formation time $\tau$ is allowed for the fireball fluid, during which the matter of the fluid propagates without interactions. The formation time is associated with a finite time of string formation. It is similarly incorporated in kinetic transport models such as UrQMD [19] and HSD [20].

Unlike the conventional hydrodynamics, where local instantaneous stopping of projectile and target matter is assumed, a specific feature of the 3FD is a finite stopping power resulting in a counter-streaming regime of leading baryon-rich matter. The basic idea of a 3-fluid approximation to heavy-ion collisions [14, 21] is that at each space-time point $x=(t, \mathbf{x})$ a generally nonequilibrium distribution function of baryon-rich matter, $f_{\mathrm{br}}(x, p)$, can be represented as a sum of two distinct contributions

$$
f_{\mathrm{br}}(x, p)=f_{\mathrm{p}}(x, p)+f_{\mathrm{t}}(x, p),
$$

initially associated with constituent nucleons of the projectile (p) and target $(\mathrm{t})$ nuclei. In addition, newly produced particles, populating the mid-rapidity region, are associated with a fireball (f) fluid. Therefore, the 3-fluid approximation is a minimal way to simulate the finite stopping power at high incident energies.

The above assumptions are implemented into the formulation of the 3FD model as follows [6]. There is a set of continuity equations (reflecting the baryon number conservation)

$$
\partial_{\mu} J_{\alpha}^{\mu}(x)=0,
$$

for $\alpha=\mathrm{p}$ and t, where $J_{\alpha}^{\mu}=n_{\alpha} u_{\alpha}^{\mu}$ is the baryon current defined in terms of baryon density $n_{\alpha}$ and hydrodynamic 4 -velocity $u_{\alpha}^{\mu}$ normalized as $u_{\alpha \mu} u_{\alpha}^{\mu}=1$. Eq. (2) implies that there is no baryon-charge exchange between p- and t-fluids, as well as that the baryon current of the fireball fluid is identically zero, $J_{\mathrm{f}}^{\mu}=0$. Equations of the energymomentum exchange between fluids are formulated in terms of energy-momentum tensors $T_{\alpha}^{\mu \nu}$ of the fluids

$$
\begin{aligned}
\partial_{\mu} T_{\mathrm{p}}^{\mu \nu}(x) & =-F_{\mathrm{p}}^{\nu}(x)+F_{\mathrm{fp}}^{\nu}(x), \\
\partial_{\mu} T_{\mathrm{t}}^{\mu \nu}(x) & =-F_{\mathrm{t}}^{\nu}(x)+F_{\mathrm{ft}}^{\nu}(x), \\
\partial_{\mu} T_{\mathrm{f}}^{\mu \nu}(x) & =-F_{\mathrm{fp}}^{\nu}(x)-F_{\mathrm{ft}}^{\nu}(x) \\
& +\int d^{4} x^{\prime} \delta^{4}\left(x-x^{\prime}-U_{F}\left(x^{\prime}\right) \tau\right)
\end{aligned}
$$

$$
\times\left[F_{\mathrm{p}}^{\nu}\left(x^{\prime}\right)+F_{\mathrm{t}}^{\nu}\left(x^{\prime}\right)\right],
$$

where the $F_{\alpha}^{\nu}$ are friction forces originating from interfluid interactions. $F_{\mathrm{p}}^{\nu}$ and $F_{\mathrm{t}}^{\nu}$ in Eqs. (3)-(4) describe energy-momentum loss of the baryon-rich fluids due to their mutual friction. A part of this loss $\left|F_{\mathrm{p}}^{\nu}-F_{\mathrm{t}}^{\nu}\right|$ is transformed into thermal excitation of these fluids, while another part $\left(F_{\mathrm{p}}^{\nu}+F_{\mathrm{t}}^{\nu}\right)$ gives rise to particle production into the fireball fluid (see Eq. (5D)). $F_{\mathrm{fp}}^{\nu}$ and $F_{\mathrm{ft}}^{\nu}$ are associated with friction of the fireball fluid with the p- and t-fluids, respectively. Here $\tau$ is the formation time, and

$$
U_{F}^{\nu}\left(x^{\prime}\right)=\frac{u_{\mathrm{p}}^{\nu}\left(x^{\prime}\right)+u_{\mathrm{t}}^{\nu}\left(x^{\prime}\right)}{\left|u_{\mathrm{p}}\left(x^{\prime}\right)+u_{\mathrm{t}}\left(x^{\prime}\right)\right|}
$$

is a free-propagating 4-velocity of the produced fireball matter. Accordingly to Eq. (5), this matter gets formed only after the time span $U_{F}^{0} \tau$ upon the production, and in different space point $\mathbf{x}^{\prime}-\mathbf{U}_{F}\left(x^{\prime}\right) \tau$, as compared to the production point $\mathbf{x}^{\prime}$.

The nucleon-nucleon cross sections at high energies are strongly forward-backward peaked. This fact, which originally served as justification for subdividing baryonic matter into target and projectile fluids, was used in 22. to estimate the friction forces, $F_{\mathrm{p}}^{\nu}$ and $F_{\mathrm{t}}^{\nu}$, proceeding from only $N N$ elastic scattering. Later these friction forces were calculated 23] based on (both elastic and inelastic) experimental inclusive proton-proton cross sections. In the present calculations the following form of the projectile-target friction is used

$$
F_{\alpha}^{\nu}=\vartheta^{2} \rho_{\mathrm{p}}^{\xi} \rho_{\mathrm{t}}^{\xi}\left[\left(u_{\alpha}^{\nu}-u_{\bar{\alpha}}^{\nu}\right) D_{P}+\left(u_{\mathrm{p}}^{\nu}+u_{\mathrm{t}}^{\nu}\right) D_{E}\right],
$$

$\alpha=\mathrm{p}$ or $\mathrm{t}, \overline{\mathrm{p}}=\mathrm{t}$ and $\overline{\mathrm{t}}=\mathrm{p}$. Here, $\rho_{\alpha}^{\xi}$ denotes a kind of "scalar density" of the p- and t-fluids (see below),

$$
D_{P / E}=m_{N} V_{\mathrm{rel}}^{\mathrm{pt}} \sigma_{P / E}\left(s_{\mathrm{pt}}\right),
$$

where $m_{N}$ is the nucleon mass, $s_{\mathrm{pt}}=m_{N}^{2}\left(u_{\mathrm{p}}^{\nu}+u_{\mathrm{t}}^{\nu}\right)^{2}$, $V_{\text {rel }}^{\mathrm{pt}}=\left[s_{\mathrm{pt}}\left(s_{\mathrm{pt}}-4 m_{N}^{2}\right)\right]^{1 / 2} / 2 m_{N}^{2}$ is the mean relative velocity of the p- and t-fluids, and $\sigma_{P / E}\left(s_{\mathrm{pt}}\right)$ are determined in terms of nucleon-nucleon cross sections integrated with certain weights (see [14, 16, 23] for details):

$$
\begin{aligned}
& \sigma_{P}\left(s_{\mathrm{pt}}\right)=\int_{\theta_{\mathrm{cm}<\pi / 2}} d \sigma_{N N \rightarrow N X}\left(1-\cos \theta_{\mathrm{cm}} \frac{p_{\text {out }}}{p_{\text {in }}}\right), \\
& \sigma_{E}\left(s_{\mathrm{pt}}\right)=\int_{\theta_{\mathrm{cm}<\pi / 2}} d \sigma_{N N \rightarrow N X}\left(1-\frac{E_{\text {out }}}{E_{\text {in }}}\right) .
\end{aligned}
$$

Here the integration is restricted to the forward hemisphere $\left(\theta_{\mathrm{cm}}<\pi / 2\right)$ of the center-of-mass scattering angles $\theta_{\mathrm{cm}}, p_{\mathrm{in}}=\left(s_{\mathrm{pt}} / 4-m_{N}^{2}\right)^{1 / 2}$ and $E_{\mathrm{in}}=s_{\mathrm{pt}}^{1 / 2} / 2$ are the in-coming momentum and energy of the nucleon in the NN c.m. frame, respectively, and $p_{\text {out }}$ and $E_{\text {out }}$ are the corresponding out-coming quantities. $\sigma_{P}\left(s_{\mathrm{pt}}\right)$ is nonzero at any physical $s_{\mathrm{pt}}$, as it is seen from Eq. (9). At the same time, the $\sigma_{E}\left(s_{\mathrm{pt}}\right)$ quantity, which is responsible for the fireball production, is zero for $s_{\mathrm{pt}}$ below the inelastic threshold. The overall $\vartheta^{2}$ factor in Eq. (7) controls 
unification of p- and t-fluid into a single one, when their relative velocity gets small enough (for details see [6]).

The above friction (7) is a certain extension of that derived in 23]. The original derivation [23] was performed under assumption that baryon-rich fluids consist of only nucleons, and only proton-proton cross sections were used in (9) and (10). The extension is required because the original derivation [23] does not take into account:

(i) various mesonic and baryonic species produced in the collision

(ii) possible multiparticle interactions which are quite probable in the dense medium,

(iii) possible medium modifications of cross sections and effective masses, and

(iv) quark and gluon interactions, if deconfinement occurs.

In view of these uncertainties, it is reasonable to make provision for tuning the above friction. For this purpose, tuning factors $\xi\left(s_{p t}\right)$ in the scalar densities of the p- and t-fluids are introduced

$$
\begin{aligned}
\rho_{\alpha}^{\xi}\left(s_{p t}\right) & =\left(\rho_{\alpha}^{\text {bar. }}+\frac{2}{3} \rho_{\alpha}^{\text {mes. }}\right) \xi_{h}\left(s_{p t}\right) \\
& +\frac{1}{3}\left(\rho_{\alpha}^{q}+\rho_{\alpha}^{g}\right) \xi_{q}\left(s_{p t}\right),
\end{aligned}
$$

where $\rho_{\alpha}^{\text {bar. }}, \rho_{\alpha}^{\text {mes. }}, \rho_{\alpha}^{q}$ and $\rho_{\alpha}^{g}$ are scalar densities of all baryons, all mesons, quarks and gluons, respectively, defined in the conventional way. This quantities are supplied together with EoS. Factors like $2 / 3$ and $1 / 3$ in Eq. (11) take into account the assumed scaling of cross sections in accordance with the naive valence-quark counting. In view of above mentioned uncertainties of the estimated friction, in Eq. (11) different tuning factors are introduced for hadronic and quark-gluon phases: $\xi_{h}$ and $\xi_{q}$, respectively.

The friction between baryon-rich fluids was fitted to reproduce the stopping power observed in proton rapidity distributions for each EoS. The results, together with those for formation time $\tau$ and freeze-out energy density $\varepsilon_{\mathrm{f} r z}$, are summarized as follows:

- Hadronic EoS with incompressibility $K=190 \mathrm{MeV}$ [7] (hadr. EoS):

$$
\begin{aligned}
& \xi_{h}^{2}(s)=1+\left[\ln \left(\frac{s^{1 / 2}}{2 m_{N}}\right)\right]^{1 / 4}, \\
& \tau=2 \mathrm{fm} / \mathrm{c}, \quad \varepsilon_{\mathrm{f} r z}=0.4 \mathrm{GeV} / \mathrm{fm}^{3} .
\end{aligned}
$$

The $\xi_{q}$ factor is not applicable here because of the pure hadronic nature of the EoS. The incompessibility $K=190 \mathrm{MeV}$ is also chosen on the condition of the best reproduction of available data.

- EoS with the first-order deconfinement transition [12] (2-phase EoS):

$$
\begin{array}{cc}
\xi_{h}^{2}(s)=1, & \xi_{q}^{2}(s)=60 \frac{4 m_{N}^{2}}{s}, \\
\tau=0.17 \mathrm{fm} / \mathrm{c}, \quad \varepsilon_{\mathrm{fr} z}=0.4 \mathrm{GeV} / \mathrm{fm}^{3} .
\end{array}
$$

- EoS with crossover deconfinement transition 12 (crossover EoS):

$$
\begin{array}{cc}
\xi_{h}^{2}(s)=1, & \xi_{q}^{2}(s)=200 \frac{4 m_{N}^{2}}{s}, \\
\tau=0.17 \mathrm{fm} / \mathrm{c}, \quad & \varepsilon_{\mathrm{f} r z}=0.4 \mathrm{GeV} / \mathrm{fm}^{3} .
\end{array}
$$

Within hadronic scenario (hadr. EoS) the friction has to be enhanced in order to reproduce the baryon stopping at high energies, $E_{l a b} \geq 10 \mathrm{~A} \mathrm{GeV}$. Though such a enhancement is admissible in view of above mentioned uncertainties, the value of the enhancement looks suspiciously high. Indeed, at $\sqrt{s_{N N}}=17.3 \mathrm{GeV}$, i.e. at the top SPS energy, $\xi_{h}^{2}=2.2$.

At scenarios with deconfinement transitions there is no need to modify the hadronic friction. This can be considered as an indirect argument in favor of such scenarios. At the same time, the quark-gluon modification factor $\xi_{q}^{2}$ decreases with the energy rise, which is in agreement with our expectations that the quark-gluon friction should get weaker at high energies because of approaching to regime of the asymptotic freedom.

Freeze-out was performed accordingly to the procedure described in [6] and in more detail in [24, 25]. The baryon stopping turns out to be only moderately sensitive to the freeze-out energy density $\varepsilon_{\mathrm{f} r z}$. The freeze-out energy density was chosen mostly on the condition of the best reproduction of secondary particles yields.

The formation time $\tau$ also affects the baryon stopping, especially at high incident energies (top SPS and higher ones), when the fireball fluid is well developed. In fact, $\tau$ reduces the effect of the friction between the fireball and baryon-rich fluids. The larger $\tau$ is, the later this friction starts to act and hence the weaker effect is produced by this friction. Therefore, the fitted value of $\tau$ is essentially related to the strength of the fireball-baryon-rich friction.

There are other full-scale (i.e. $(3+1)$-dimensional) approaches to modeling nuclear collisions, which take into account the deconfinement trasition. These have their advantages, as well as disadvantages as compared to the 3 FD model. The conventional hydrodynamical model of Refs. [26, 27] does such simulations in a very similar way but without taking into account incomplete stopping of colliding nuclei at the initial stage of the reaction. Therefore, such kind of simulations are justified only at moderately high energies. Another class of fluid models uses (hadronic) kinetic codes to "cook" the initial fireball which is subsequently considered within the hydro simulation with possible deconfinement transitions [28 31]. Such approaches disregard effects of deconfinement transitions at the stage of inter-penetration of colliding nuclei, and hence cannot be used for analysis of the baryon stopping, which is the prime goal of this article. Kinetic models with a deconfinement transition, i.e. A MultiPhase Transport model [32] and a more consistent model of Parton-Hadron-String Dynamics [33], overcome this problem. Moreover, they avoid the problem of freezeout inherent in hydrodynamic models. However, kinetic 
models are able to treat only a crossover transition. The first-order transition remains beyond the scope of kinetics. Contrary to other hydrodynamic approaches, the 3FD model can treat a deconfinement transition at the initial stage of the collision with due account of incomplete stopping of colliding nuclei, though in essentially rougher approximation than that in kinetic models. At the same time the 3FD model is able to work with the first-order transition unlike kinetic models.

In the present run of computations, higher incident energies were reached as compared with previous runs. This became possible because of implementation of an adaptive grid in the code. The size of the cell is made gradually larger with the expansion of the system proceeded. Thus, when the system occupies the larger space, the code does not require a larger number of cells and, hence, a higher RAM memory. The adaptive grid does not make the accuracy worse since spatial distributions become smoother at the expansion, that relaxes requirements on the grid step. The adaptive grid made possible computations up to $62.4 \mathrm{GeV}$ incident energy in terms of the c.m. nucleon-nucleon energy, i.e. $\sqrt{s_{N N}}$. However, results for the top energy of $62.4 \mathrm{GeV}$ are still not quite accurate, since an accurate computation requires unreasonably high memory and CPU time. This should be kept in mind when results for this energy are displayed.

\section{EQUATIONS OF STATE}

Figure 1 illustrates differences between three considered EoS's. The deconfinement transition makes a EoS softer at high densities. The 2-phase EoS is based on the Gibbs construction, taking into account simultaneous conservation baryon and strange charges. However, the displayed result looks very similar to the Maxwell construction, corresponding to conservation of only baryon charge, with the only difference that the plateau is slightly tilted, which is practically invisible. This invisible slope of the plateau results from plotting the pressure at the additional condition of strange density being equal to zero rather than at constant strange chemical potential. Application the Gibbs construction in hydrodynamical simulations silently assumes that the inter-phase equilibration in the mixed-phase region is faster than the hydrodynamical evolution.

The 2-phase and crossover EoS's still differ even at very high densities. The latter means that the crossover transition constructed in Ref. [12] is very smooth. The hadronic fraction survives up to very high densities. In particular, this is seen from Fig. 2, the fraction of the quark-gluon phase $\left(W_{Q G P}\right)$ reaches value of 0.5 only at very high energy densities. In this respect, this version of the crossover EoS certainly contradicts results of the lattice QCD calculations, where a fast crossover, at least at zero chemical potential, was found [34]. Therefore, a true EoS is somewhere in between the crossover and 2-phase EoS's of Ref. [12].

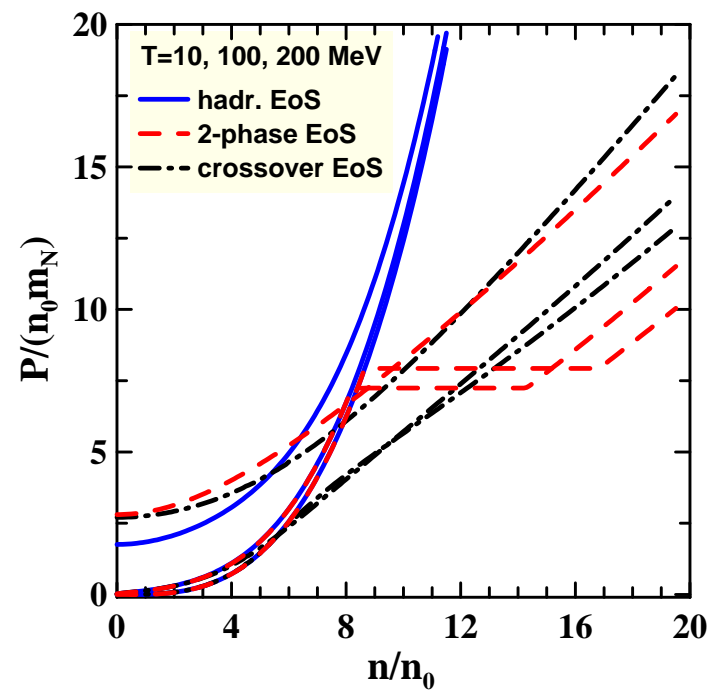

FIG. 1: (Color online) Pressure scaled by the product of normal nuclear density $\left(n_{0}=0.15 \mathrm{fm}^{-3}\right)$ and nucleon mass $\left(m_{N}\right)$ versus baryon density scaled by the normal nuclear density for three considered equations of state. Results are presented for three different temperatures $T=10,100$ and $200 \mathrm{MeV}$ (bottom-up for corresponding curves).

Figure 2 demonstrates that the onset of the deconfinement transition in the calculations happens at topAGS-low-SPS energies. Similarly to that it has been done in [35], the figure displays dynamical trajectories of the matter in the central box placed around the origin $\mathbf{r}=(0,0,0)$ in the frame of equal velocities of colliding nuclei: $|x| \leq 2 \mathrm{fm},|y| \leq 2 \mathrm{fm}$ and $|z| \leq \gamma_{c m} 2 \mathrm{fm}$, where $\gamma_{c m}$ is Lorentz factor associated with the initial nuclear motion in the c.m. frame. Initially, the colliding nuclei are placed symmetrically with respect to the origin $\mathbf{r}=(0,0,0), z$ is the direction of the beam. At a given density $n_{B}$, the zero-temperature compressional energy, $\varepsilon\left(n_{B}, T=0\right)$, provides a lower bound on the energy density $\varepsilon$, so the accessible region is correspondingly limited. In the case of the crossover EoS only the region of the mixed phase between $W_{Q G P}=0.1$ and $W_{Q G P}=$ 0.5 is displayed, since in fact the mixed phase occupies the whole $\left(\varepsilon-n_{B}\right)$ region. The $\varepsilon-n_{B}$ representation is chosen because these densities are dynamical quantities and, therefore, are suitable to compare calculations with different EoS's.

Only expansion stages of the evolution are displayed, where the matter in the box is already thermally equilibrated, as a rule. The exceptions are central collisions at $\sqrt{s_{N N}}=27$ and $39 \mathrm{GeV}$, in which the matter in the box is not still thermalized in the beginning of the expansion stage. This non-equilibrium stage of the expansion is displayed by dashed lines in Fig. 2, The criterion of the thermalization is equality of longitudinal and transverse pressures in the box with the accuracy better than $10 \%$. Evolution proceeds from the top point of the trajectory downwards. Symbols mark the time intervals along 

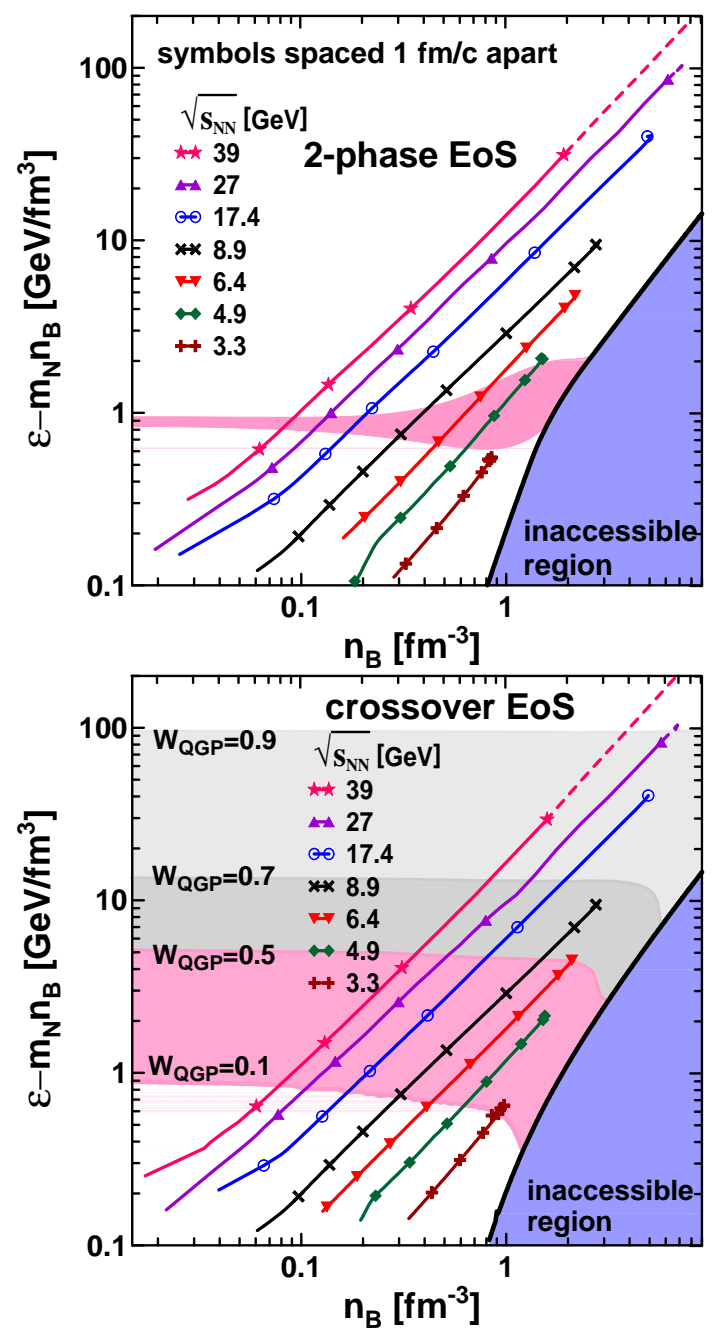

FIG. 2: (Color online) Dynamical trajectories of the matter in the central box of the colliding nuclei $\left(4 \mathrm{fm} \times 4 \mathrm{fm} \times \gamma_{c m} 4 \mathrm{fm}\right)$, where $\gamma_{c m}$ is the Lorentz factor associated with the initial nuclear motion in the c.m. frame, for central collisions of $\mathrm{Au}+\mathrm{Au}$ at $\sqrt{s_{N N}}=3.3,4.9,27$ and $39 \mathrm{GeV}(b=2 \mathrm{fm})$, and $\mathrm{Pb}+\mathrm{Pb}$ at $\sqrt{s_{N N}}=6.4,8.9$ and $17.4(b=2.4 \mathrm{fm})$. The trajectories are plotted in terms of baryon density $\left(n_{B}\right)$ and the energy density minus $n_{B}$ multiplied by the nucleon mass $\left(\varepsilon-m_{N} n_{B}\right)$. Only expansion stages of the evolution are displayed. Symbols on the trajectories indicate the time rate of the evolution: time span between marks is $1 \mathrm{fm} / \mathrm{c}$. For the 2-phase EoS (upper panel) the shadowed "mixed phase" region is located between the borders, where the QGP phase start to raise $\left(W_{Q G P}=0\right)$ and becomes completely formed $\left(W_{Q G P}=1\right)$. For the crossover EoS (lower panel) the displayed borders correspond to values of the QGP fraction $W_{Q G P}=0.1,0.5,0.7$ and 0.9 . Inaccessible region is restricted by $\varepsilon\left(n_{B}, T=0\right)-m_{N} n_{B}$ from above.

the trajectory. Subtraction of the $m_{N} n_{B}$ term is taken for the sake of suitable representation of the plot. The size of the box was chosen to be large enough that the amount of matter in it can be representative to conclude on the onset of deconfinement and to be small enough to consider the matter in it as a homogeneous medium.

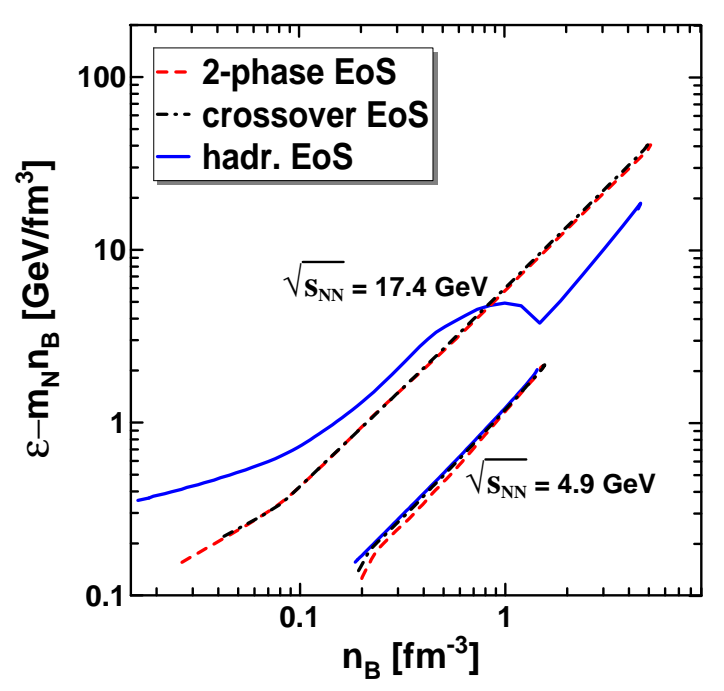

FIG. 3: (Color online) The same as in Fig. 2 but for trajectories for all three different EoS's within the same frame.

Nevertheless, the matter in the box still amounts to a minor part of the total matter of colliding nuclei. Therefore, only the minor part of the total matter undergoes the deconfinement transition at $10 \mathrm{~A} \mathrm{GeV}$ energy.

As seen, the deconfinement transition starts at the top AGS energies in both cases. It gets practically completed at low SPS energies in the case of the 2-phase EoS. In the crossover scenario it lasts till very high incident energies.

The trajectories for different EoS's are very similar at lower incident energies, as it seen from Fig. 3 At higher energies trajectories for deconfinement scenarios remain very similar, while the hadronic-EoS trajectories differ from those mentioned above and exhibit a peculiar behavior. It happens because of a long (as compared with the interpenetration time of colliding nuclei) formation time of the fireball fluid, see (13). At the first stage the expansion proceeds when the fireball fluid has not been formed yet. Then the formation starts and the energy density (but not the baryon density) even slightly rises. When the formation is practically completed, the trajectory returns to its normal evolution - downward in energy and baryon densities.

\section{PROTON AND NET-PROTON RAPIDITY DISTRIBUTIONS}

A direct measure of the baryon stopping is the netbaryon (i.e. baryons-minus-antibarions) rapidity distribution. However, since experimental information on neutrons is unavailable, we have to rely on net-proton (i.e. proton-minus-antiproton) data. Presently there exist experimental data on proton (or net-proton) rapidity spectra at AGS [36 39] and SPS [40 44] energies. These data were analyzed within various models [6, 8, 26, 27, 29, 33, 45 49]. The most extensive analysis has been done in [6, 47]. Here I would like to repeat 

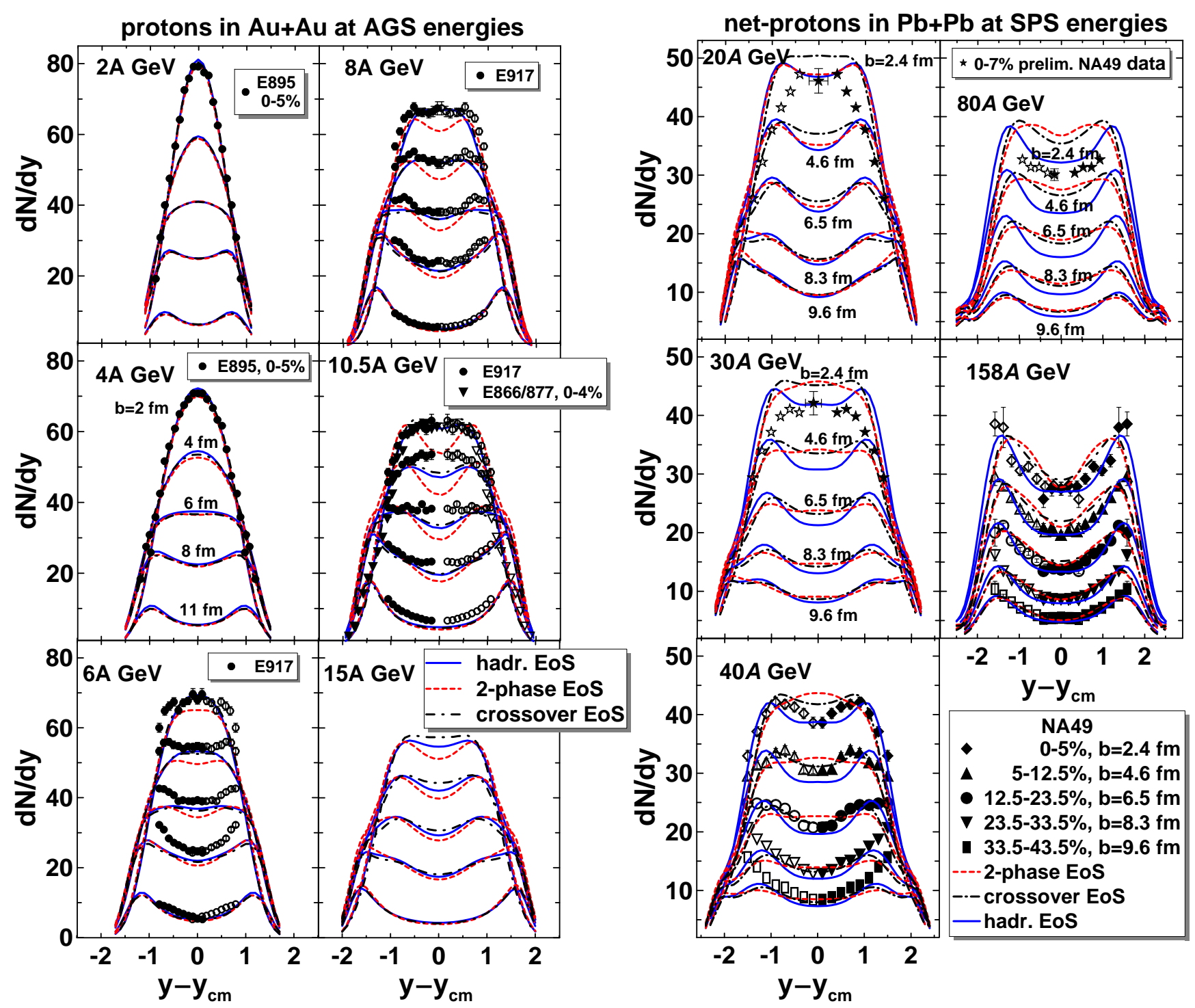

FIG. 4: (Color online) Rapidity spectra of protons (for AGS energies, left block of panels) and net-protons ( $p-\bar{p}$ ) (for SPS energies, right block of panels) from collisions of $\mathrm{Au}+\mathrm{Au}(\mathrm{AGS})$ and $\mathrm{Pb}+\mathrm{Pb}(\mathrm{SPS})$ calculated within three considered scenarios. Experimental data are from collaborations E895 [36], E877 [37], E917 [38], E866 [39], and NA49 [40 44]. The percentage shows the fraction of the total reaction cross section, corresponding to experimental selection of events. For the E917 data [38] these are $0-5 \%, 5-12 \%, 12-23 \%, 23-39 \%$ and $39-81 \%$. Feedback of weak decays into $p$ and $\bar{p}$ yields is disregarded.

this analysis. The motivation is to perform simulations with different EoS's within the same dynamical model, i.e. the 3FD model, in order to reveal differences produced by different scenarios.

Figure 4 presents calculated rapidity distributions of protons (for AGS energies) and net-protons (for SPS energies) and their comparison with available data. Notice that difference between protons and net-protons is negligible at the AGS energies. At the top AGS energy of $10 \mathrm{~A}$ $\mathrm{GeV}$ their difference is $0.03 \%$ at the midrapidity, see compilation of experimental data in Ref. [50]. Contribution of weak decays of strange hyperons into proton yield was disregarded in accordance with mesurement conditions of the NA49 collaboration. At the AGS energies the con- tribution of weak decays is negligible. Correspondence between the fraction of the total reaction cross section related to a data set and a mean value of the impact parameter was read off from the paper [51] in case of NA49 data. For $\mathrm{Au}+\mathrm{Au}$ collisions it was approximately estimated proceeding from geometrical considerations.

As seen from Fig. 4, at lower AGS energies all EoS's predict the same results, since at these energies only hadronic parts of all EoS's are relevant. Results of the 2-phase EoS start to differ from those of the hadr. and crossover EoS's beginning from $6 \mathrm{~A} \mathrm{GeV}$ and first in central collisions. At higher energies this difference extends to more peripheral collisions. Unlike other scenarios, the 2-ph.-EoS distributions exhibit a dip at midrapidity even 

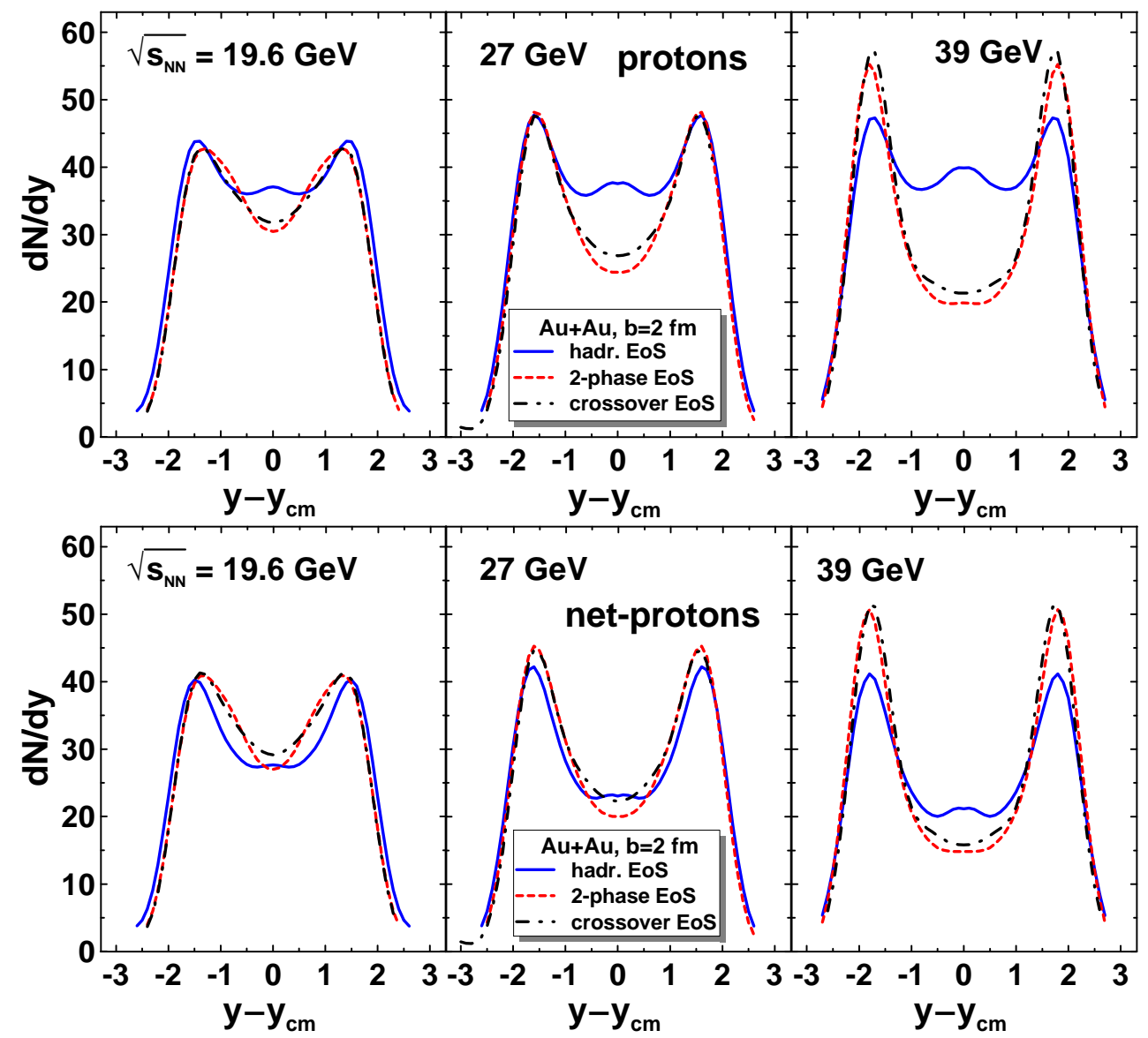

FIG. 5: (Color online) Rapidity spectra of protons (upper panel) and net-protons (lower panel) from central collisions of Au+Au $(b=2 \mathrm{fm})$ at low RHIC energies. Feedback of weak decays into $p$ and $\bar{p}$ yields is taken into account.

in central collisions. This dip contradicts the available experimental data and is very robust: variation of the model parameters (14) and (15) in a wide range does not remove this dip. Therefore, it is a direct consequence of the onset of the first-order phase transition, which starts precisely at these energies in the 2-phase scenario, see Fig. 2] Calculations within one-fluid (i.e. conventional hydrodynamics) [26, 27] confirm this conclusion. This dip survives even in one-fluid calculations involving the 1st-order phase transition in spite of immediate baryon stopping inherent in the one-fluid model. Notice that one-fluid calculations without deconfinement transition manifest a "normal" (for the one-fluid model) result, i.e. no dip. Accordingly to analysis of Ref. [26] this dip is a consequence of larger pressure gradients in the longitudinal direction developed in the deconfinement-transition scenario. In 3FD calculations, this dip transforms into midrapidity peak at higher energies $(30 \mathrm{~A} \mathrm{GeV}$ and $40 \mathrm{~A}$ $\mathrm{GeV})$. With further energy rise $\left(E_{l a b}>40 A \mathrm{GeV}\right)$ the midrapidity peak again turns into a dip, see also Fig. 5 The latter dip is already a normal behavior which results from incomplete stopping of baryons and takes place at arbitrary high energies.

As has been already mentioned, the behavior "peak- dip-peak-dip" in central collisions within the 2-phase-EoS scenario is very robust with respect to variation of the model parameters (14) and (15) in a wide range. It certainly disagrees with data at $8 A \mathrm{GeV}, 10 A \mathrm{GeV}$ and $40 A$ $\mathrm{GeV}$ energies. It also disagrees with data at $20 \mathrm{~A} \mathrm{GeV}$ and $30 \mathrm{~A} \mathrm{GeV}$. However, the latter data have preliminary status, and hence it is too early to draw any conclusions from comparison with them. This behavior is in contrast with that for the hadronic-EoS scenario, where the form of distribution in central collisions gradually evolves from peak at the midrspidity to a dip. The case of the crossover EoS is intermediate. One could conclude in favor of a weak wiggle, since the distributions at $10 \mathrm{~A}$ and $15 \mathrm{~A} \mathrm{GeV}$ exhibit a shallow dip while at $20 \mathrm{~A} \mathrm{GeV}$ looks like a plateau.

Beginning from $158 \mathrm{~A} \mathrm{GeV}$ to higher incident energies (see Fig. 5) predictions of different scenarios for the netproton distributions remain quite similar, at the expense of the substantial enhancement of the hadronic friction in the case of the hadronic EoS, see Eq. (12). At the same time difference of proton spectra increase with the energy rise. In calculations for energies above $158 \mathrm{~A} \mathrm{GeV}$ contribution of weak decays into proton and net-proton yields were taken in to account in accordance of experimental 

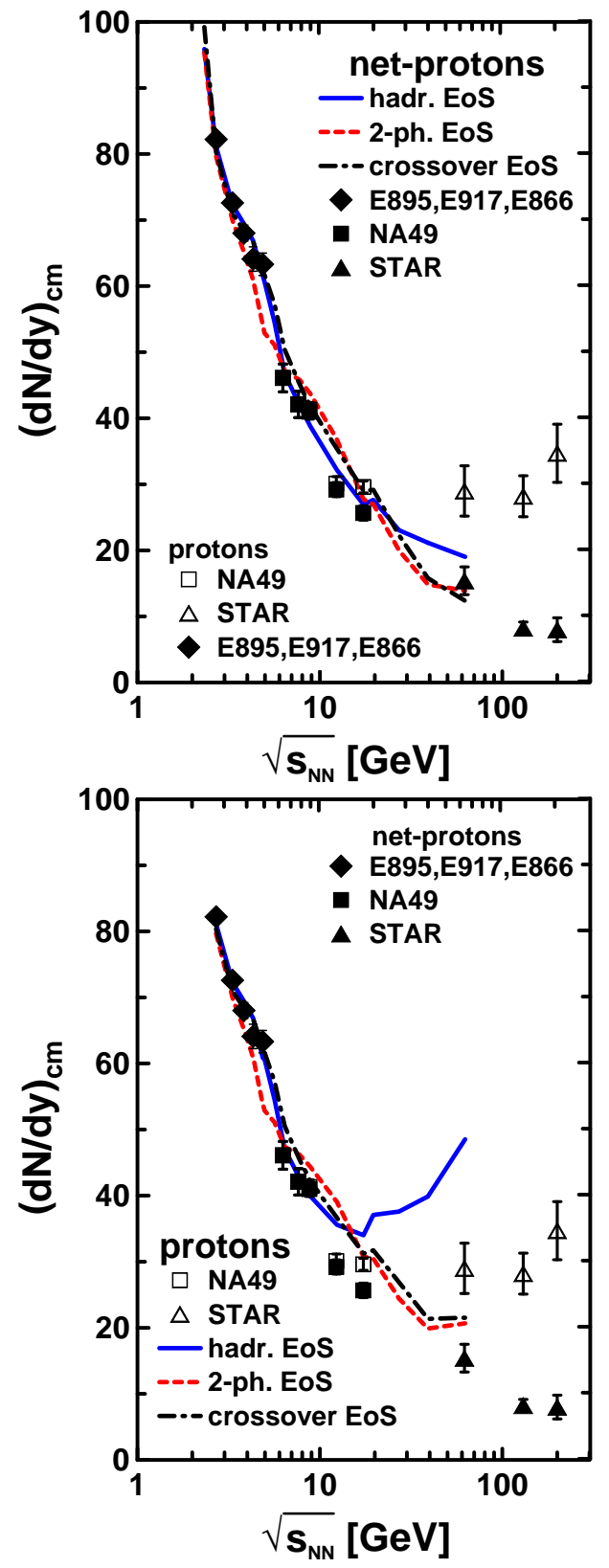

FIG. 6: (Color online) Midrapidity value of the net-proton (upper panel) and proton (lower panel) rapidity spectrum from central Au+Au (at AGS and RHIC energies, $b=2 \mathrm{fm}$ ) and $\mathrm{Pb}+\mathrm{Pb}$ (at SPS energies, $b=2.4 \mathrm{fm}$ ) collisions as a function of the incident center-of-mass energy for three considered EoS's. Experimental data are from collaborations E895 36], E877 [37], E917 38], E866 [39], NA49 [40 44] and STAR [52].

procedure of STAR and PHENIX collaborations.

Comparison with available data indicate certain preference of the crossover EoS, though the crossover scenario does not perfectly reproduce the data either. Predictions of different scenarios for net-protons diverge to the largest extent in the energy region $8 A \mathrm{GeV} \leq E_{l a b} \leq$ $40 A \mathrm{GeV}$. Unfortunately data at $20 A$ and $30 A \mathrm{GeV}$ still have preliminary status and disagree with any considered

scenario. Updated experimental results at energies $20 \mathrm{~A}$ and $30 A \mathrm{GeV}$ are badly needed to pin down the preferable EoS and to check a trend of the "peak-dip-peak-dip" irregularity in the net-proton rapidity distributions.

Preference of the deconfinement-transition scenarios is seen at incident energies above the top SPS one, see Fig. 6. where midrapidity values of the net-proton and proton rapidity spectra from central collisions of $\mathrm{Au}+\mathrm{Au}$ (at AGS and RHIC energies) and $\mathrm{Pb}+\mathrm{Pb}$ (at SPS energies) are plotted as functions of the incident center-of-mass energy. In Fig. 6 the midrapidity values are displayed in a wider energy range. I would like to remind that results for top calculated energy of $\sqrt{s_{N N}}=62.4 \mathrm{GeV}$ are very approximate, since a more accurate computation requires unreasonably high memory and CPU time. As seen, a visible difference between net-protons and proton data, as well as between predictions of hadronic EoS and EoS's with deconfinement transitions starts only at RHIC energies. At these energies the hadronic scenario certainly overestimates the proton midrapidity density.

\section{ANALYSIS OF "PEAK-DIP-PEAK-DIP" IRREGULARITY}

Preliminary results of the above-discussed "peak-dippeak-dip" irregularity have been already reported in Refs. [53, 54]. There the friction forces for the 2-phase and crossover scenarios were poorly tuned and hence the corresponding simulations poorly reproduced available experimental data. Therefore, conclusions were based on a certain trend of the results of simulations. Here I present calculations with thoroughly tuned friction forces in the quark-gluon phase, which made it possible to reasonably (and often better than in the hadronic scenario) reproduce a great number of observables in a wider (than before [53, 54] ) incident energy range $2.7 \mathrm{GeV} \leq \sqrt{s_{N N}} \leq$ $39 \mathrm{GeV}$.

In order to quantify the "peak-dip-peak-dip" irregularity, it is useful to make use of the method proposed in Refs. [13, 53, 54]. For this purpose the data on netproton rapidity distributions are fitted by a simple formula

$$
\begin{aligned}
\frac{d N}{d y} & =a\left(\exp \left\{-\left(1 / w_{s}\right) \cosh \left(y-y_{c m}-y_{s}\right)\right\}\right. \\
& \left.+\exp \left\{-\left(1 / w_{s}\right) \cosh \left(y-y_{c m}+y_{s}\right)\right\}\right)
\end{aligned}
$$

where $a, y_{s}$ and $w_{s}$ are parameters of the fit. The form (18) is a sum of two thermal sources shifted by $\pm y_{s}$ from the midrapidity. The width $w_{s}$ of the sources can be interpreted as $w_{s}=($ temperature $) /($ transverse mass $)$, if we assume that collective velocities in the sources have no spread with respect to the source rapidities $\pm y_{s}$.

The above fit has been done by the least-squares method. Data were fitted in the rapidity range $\mid y-$ $y_{c m} \mid / y_{c m}<0.7$. The choice of this range is dictated by the data. As a rule, the data are available in this rapidity range, sometimes the data range is even more narrow 


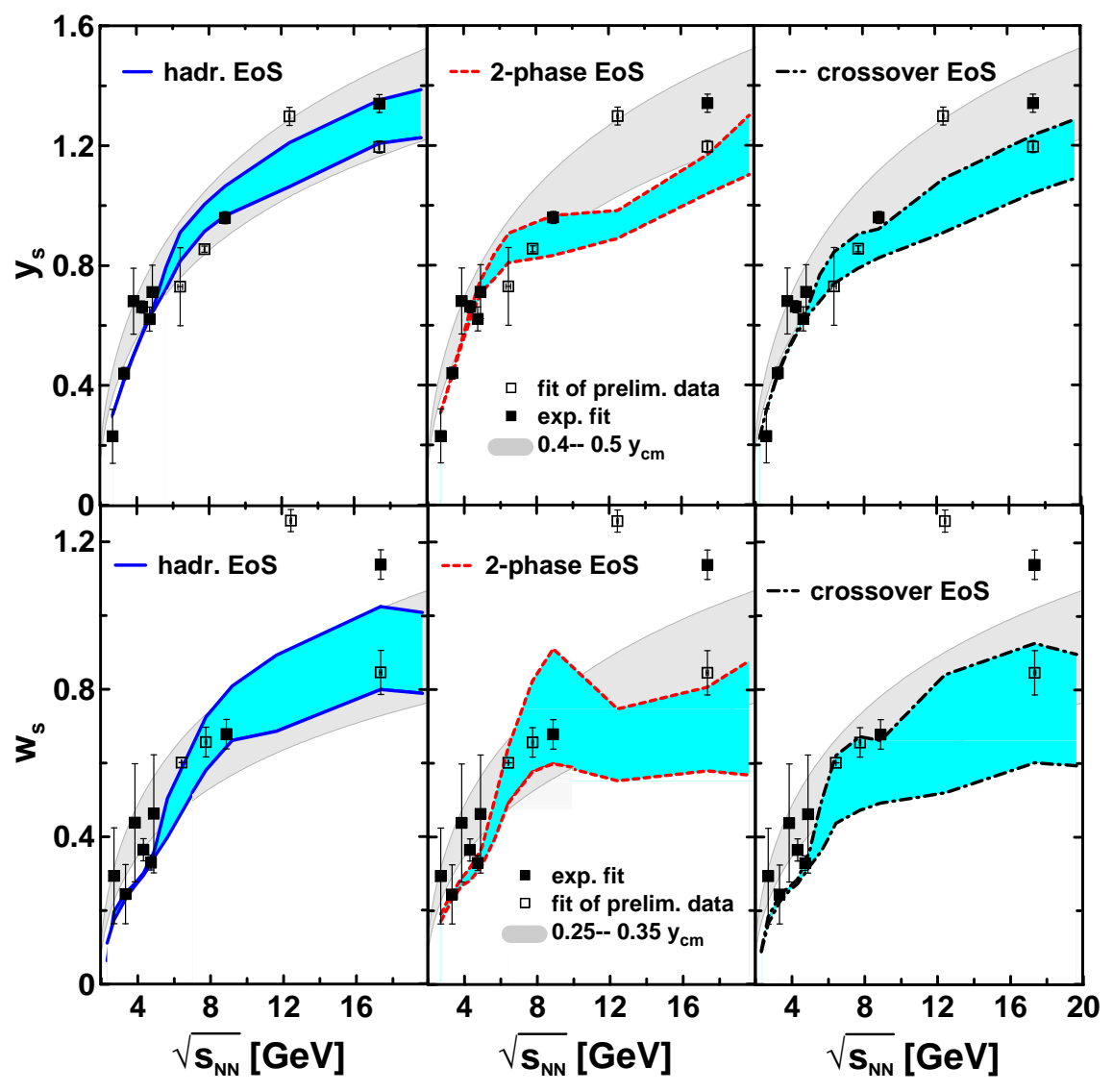

FIG. 7: (Color online) Parameters of fit (18), $y_{s}$ (upper raw of panels) and $w_{s}$ (lower raw of panels), deduced from analysis of experimental data of net-proton rapidity distributions (points with error bars), as well as from that of results of $3 \mathrm{FD}$ simulations with different EoS's (shaded areas between respective lines). Fits of preliminary experimental data are displayed by open symbols, while those for confirmed data - by filled symbols. Lines, restricting shaded areas for different EoS's, are obtained by fits within different rapidity ranges: upper curves in the range of $\left|y-y_{c m}\right| / y_{c m}<0.5$, lower curves in $\left|y-y_{c m}\right| / y_{c m}<0.7$. Grey bands in all the panels indicate the areas: between $0.25 y_{\mathrm{cm}}$ and $0.35 y_{\mathrm{cm}}$ for $w_{\mathrm{s}}$ (lower raw of panels), and $0.4 y_{\mathrm{cm}}$ and $0.5 y_{\mathrm{cm}}$ for $y_{s}$ (upper raw of panels).

( $80 A \mathrm{GeV}$ and new data at $158 A \mathrm{GeV}[\underline{44}])$. The above constraint is imposed in order to treat different data in approximately the same rapidity range. Another reason for this cut is that the rapidity range should not be too wide in order to exclude contribution of could spectators. I keep the old data at $158 \mathrm{~A} \mathrm{GeV} \mathrm{[40]} \mathrm{in} \mathrm{the} \mathrm{anal-}$ ysis because these are known in a wider rapidity range as compared with the new ones [44]. A narrow rapidity range results in large error bars of the fit. To evaluate errors of the fit parameters, I estimated the errors produced by the least-squares method, as well as performed fits in different the rapidity ranges: $\left|y-y_{\mathrm{cm}}\right| / y_{\mathrm{cm}}<0.5$ and $\left|y-y_{c m}\right| / y_{c m}<0.7$, where it is appropriate. The error bars present largest uncertainties among mentioned above.

Similar fit was applied to the calculated distributions. Since experimental data at AGS and RHIC energies were taken from $\mathrm{Au}+\mathrm{Au}$ collisions while at SPS the $\mathrm{Pb}+\mathrm{Pb}$ collisions were studied, the calculations were performed respectively for $\mathrm{Au}+\mathrm{Au}(b=2 \mathrm{fm})$ and $\mathrm{Pb}+\mathrm{Pb}(b=$ $2.4 \mathrm{fm})$ central collisions. In fact, at the same inci- dent energy the computed results for $\mathrm{Pb}+\mathrm{Pb}$ collisions at $b=2.4 \mathrm{fm}$ are very close to those for $\mathrm{Au}+\mathrm{Au}$ at $b=2$ $\mathrm{fm}$. Therefore, the related irregularity of the energy dependence of the fit parameters is negligible. Similarly to the experimental data, the fit of the computed results was also performed in two ranges, $\left|y-y_{c m}\right| / y_{c m}<0.7$ and $\left|y-y_{\mathrm{cm}}\right| / y_{\mathrm{cm}}<0.5$, in order to estimate uncertainty associated with varition of this range. This uncertainty turned out to be a dominant one in the case of computed data. Therefore, in Figs. 7 and 8 results of the fit of computed spectra are presented by shaded areas with borders corresponding to the fit ranges $\left|y-y_{\mathrm{cm}}\right| / y_{\mathrm{cm}}<0.7$ and $\left|y-y_{c m}\right| / y_{c m}<0.5$.

Parameters $y_{s}$ and $w_{s}$ deduced from the fit of experimental data exhibits no significant irregularities in their energy dependence: they monotonously rise with the energy within the error bars of the fit, see Fig. 7. Grey bands in all the panels of Fig. 7 are drawn to guide an eye. They indicate the areas: between $0.25 y_{\mathrm{cm}}$ and $0.35 y_{\mathrm{cm}}$ for $w_{s}$ (lower raw of panels), and between $0.4 y_{\mathrm{cm}}$ and $0.5 y_{\mathrm{cm}}$ for $y_{s}$ (upper raw of panels), where the ma- 


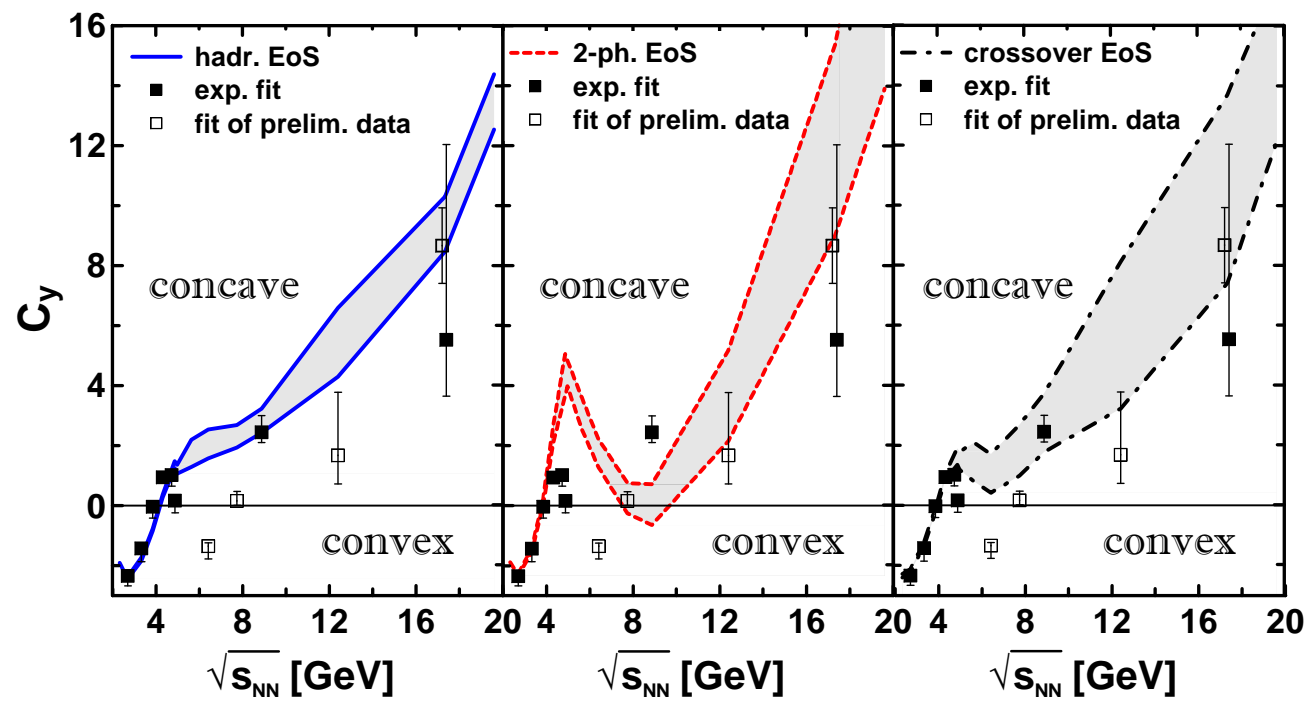

FIG. 8: (Color online) Midrapidity reduced curvature [see. Eq. [19]) of the net-proton rapidity spectrum as a function of the incident center-of-mass energy of colliding nuclei as deduced from experimental data and predicted by 3FD calculations with different EoS's. Upper borders of the shaded areas correspond to fits confined in the region of $\left|y-y_{c m}\right| / y_{c m}<0.7$, lower borders $-\left|y-y_{c m}\right| / y_{c m}<0.5$.

jor part of experimental points are located. In particular, similar (but based on different, double-gaussian fit) analysis of Ref. [55] also shows absence of any spectacular irregularities in exitation functions of these parameters. This is more so in view of the fact that the analysis of Ref. [55] was performed only at $E_{l a b} \geq 20 \mathrm{~A} \mathrm{GeV}$. The parameters deduced from the fit of distributions computed within hadronic and crossover scenarios also manifest quite monotonous behavior with incident energy. At the same time, the results of 2-phase scenario exhibit certain, however not strong, irregularity.

The representation in terms of $y_{s}$ and $w_{s}$ is not quite spectacular. These parameters are interrelated to some extent. They produce a similar effect on the rapidity distribution, especially if it is fitted in a narrow rapidity range. Therefore, it is desirable to find a single quantity which characterizes the shape of the rapidity distribution in the midrapidity range. Such a parameter is a reduced curvature of the spectrum in the midrapidity defined as follows

$$
\begin{aligned}
C_{y} & =\left(y_{c m}^{3} \frac{d^{3} N}{d y^{3}}\right)_{y=y_{c m}} /\left(y_{c m} \frac{d N}{d y}\right)_{y=y_{c m}} \\
& =\left(y_{c m} / w_{s}\right)^{2}\left(\sinh ^{2} y_{s}-w_{s} \cosh y_{s}\right) .
\end{aligned}
$$

The factor $1 /\left(y_{c m} d N / d y\right)_{y=y_{c m}}$ is introduced in order to get rid of overall normalization of the spectrum, i.e. of the $a$ parameter in terms of fit (18). The second part of Eq. (19) presents this curvature in terms of parameters of fit (18). Thus, the reduced curvature, $C_{y}$, and the midrapidity value of the distribution are two independent quantities quantifying the the spectrum in the midrapidity range. Excitation functions of $C_{y}$ deduced both from experimental data and results computed with different EoS's are displayed in Fig. 8 Fig. 8 demon- strates how the distribution shape evolves from a convex form at low incident energies to a concave form at high energies.

The irregularity in data is distinctly seen here as a zigzag irregularity in the energy dependence of $C_{y}$. Of course, this is only a hint to irregularity since this zigzag is formed only due to preliminary data of the NA49 collaboration. A remarkable observation is that the $C_{y}$ energy dependence in the first-order-transition scenario manifests qualitatively the same zigzag irregularity (middle panel of Fig. 8), as that in the data fit, while the hadronic scenario produces purely monotonous behaviour. The crossover EoS represents a very smooth transition, as mentioned above. Therefore, it is not surprising that it produces only a weak wiggle in $C_{y}$.

As it was explained in detail in Ref. [13], the "peakdip-peak-dip" irregularity is very natural in a system undergoing a phase or crossover transition. First, it is associated with the softest point of a EoS [56]. Therefore, the irregularity is weaker in the crossover scenario than in the first-order-transition one. Indeed, the softest points in the crossover EoS is less pronounced than in the firstorder-transition one [57]. There is no softest point in the hadronic EoS and hence there is no irregularity.

The second reason of this irregularity is a change in the nonequilibrium regime. The 3FD model takes into account the leading nonequilibrium of the nuclear collision associated with a finite stopping power of the nuclear matter. It simulates the finite stopping power by means of friction between three fluids. Naturally, this friction changes when deconfinement happens. In the case of the crossover scenario this change in the friction is very smooth. Therefore, it does not contribute to the irregularity. At the same time this change in the friction 
enhances the irregularity in the first-order-transition scenario. As it was demonstrated in Ref. 53], if the same friction is used in both phases, the reduced curvature calculated with the 2-phase EoS exhibits only a weak wiggle in $C_{y}$ with considerably smaller amplitude as compared with zigzag in actual calculations with different frictions in different phases. These different frictions appear quite naturally in the 3FD model. The hadronic friction was estimated in Ref. 23] and works well at lower AGS energies. Therefore, there are no reasons to modify it. The partonic friction, while not microscopically estimated, is fitted to reproduce data at high incident energies. This is a reason to believe that it is a proper choice.

It is important to emphasize that the "peak-dip-peakdip" irregularity is a signal from the hot and dense stage of the nuclear collision, rather than from the freeze-out stage as the most part of signal are.

\section{SUMMARY}

Proton and net-proton rapidity distributions in collisions of heavy nuclei $\mathrm{Au}+\mathrm{Au}$ (at AGS and RHIC energies) and $\mathrm{Pb}+\mathrm{Pb}$ (at SPS energies) were analyzed in a wide range of incident energies $2.7 \mathrm{GeV} \leq \sqrt{s_{N N}} \leq 39$ $\mathrm{GeV}$ in terms of the center-of-mass energy per nucleon pair. The analysis was done within a model of the threefluid dynamics [6] employing three different equations of state: a purely hadronic EoS [7] and two versions of EoS involving the deconfinement transition [12]. These are an EoS with the first-order phase transition and that with a smooth crossover transition. The crossover transition constructed in Ref. [12] is very smooth. The hadronic fraction survives up to very high energy densities. In this respect, this version of the crossover EoS certainly contradicts results of the lattice QCD calculations, where a fast crossover, at least at zero chemical potential, was found. Therefore, a true EoS is somewhere in between the crossover and 2-phase EoS's of Ref. [12].

Scenarios based on EoS's with deconfinement transitions have a theoretical advantage as compared to the purely hadronic one. In order to reproduce the baryon stopping at high incident energies, the friction between counter-streaming fluids have to be enhanced within the hadronic scenario as compared to its estimate based on experimental inclusive proton-proton cross sections [23]. Though such enhancement is admissible in view of uncertainties of the estimated friction, the value of the enhancement looks too high. In scenarios with deconfinement there is no need to modify the hadronic friction. This can be considered as an indirect argument in favor of such scenarios.

It was found that predictions within the first-ordertransition scenario, i.e. with the 2-phase EoS, exhibit a "peak-dip-peak-dip" irregularity in the incident energy dependence of the form of the net-proton rapidity distributions. At low energies, up to $E_{l a b}=6 \mathrm{~A} \mathrm{GeV}$, rapidity distributions for central collisions have a peak at the midrapidity, similarly to results with other EoS's. Beginning from $8 \mathrm{~A} \mathrm{GeV}$, this peak turns into a dip at the midrapidity. Then again a peak is realized, starting from $30 A \mathrm{GeV}$. With further energy rise $\left(E_{l a b}>40 A \mathrm{GeV}\right)$ the midrapidity peak again transforms into a dip, which is already a normal behavior which takes place at arbitrary high energies. This behavior is in contrast with that for the hadronic scenario, where the form of distribution in central collisions gradually evolves from peak at the midrspidity (at $E_{l a b}<10 \mathrm{~A} \mathrm{GeV}$ ) to a dip (at $E_{l a b} \gtrsim$ $10 A \mathrm{GeV})$. The case of the crossover EoS is intermediate. Only a weak wiggle of the type of "peak-dip-peak-dip" is observed in the energy range of $10 \mathrm{~A} \mathrm{GeV} \leq E_{l a b} \leq 20 \mathrm{~A}$ $\mathrm{GeV}$.

The behavior the type of "peak-dip-peak-dip" in central collisions within the 2-phase-EoS scenario is very robust with respect to variation of the model parameters in a wide range. It certainly disagrees with data at $8 A \mathrm{GeV}$, $10 A \mathrm{GeV}$ and $40 A \mathrm{GeV}$ energies. It also disagrees with data at $20 A \mathrm{GeV}$ and $30 A \mathrm{GeV}$, which have preliminary status, and hence it is too early to draw any conclusions from comparison with them.

However, the experimental data also exhibit a trend of the "peak-dip-peak-dip" irregularity in the energy range $8 A \mathrm{GeV} \leq E_{l a b} \leq 40 A \mathrm{GeV}$. Again this trend is based on preliminary data at energies of $20 A \mathrm{GeV}$ and $30 A \mathrm{GeV}$. Therefore, updated experimental results at $20 A$ and $30 A$ $\mathrm{GeV}$ are badly needed to pin down the preferable EoS and to check the trend of the "peak-dip-peak-dip" behavior in net-proton rapidity distributions. An irregularity in the baryon stopping is a signal of deconfinement occurring in the compression stage of a nuclear collision. It is a combined effect of the softest point of a EoS and a change in the nonequilibrium regime from hadronic to partonic one. It is important to emphasize that this irregularity is a signal from the hot and dense stage of the nuclear collision.

An effective method to quantify the "peak-dip-peakdip" irregularity is the analysis of the distribution shape in terms of the reduced curvature of the spectrum in the midrapidity $C_{y}$. In tems of $C_{y}$ this irregularity is distinctly seen as a zigzag irregularity in the energy dependence of $C_{y}$.

Comparison with available data, including those at RHIC energies, indicate certain preference of the crossover EoS, though the crossover-EoS scenario in the presently used version does not perfectly reproduce the data either. Predictions of different scenarios for netprotons diverge to the largest extent in the energy region $8 A \mathrm{GeV} \leq E_{l a b} \leq 40 A \mathrm{GeV}$. The preliminary data at $20 A$ and $30 A \mathrm{GeV}$ disagree with any of the considered scenarios. At incident energies above the top SPS one the hadronic scenario certainly overestimates the proton midrapidity density, while deconfinement scenarios reasonably reproduce it.

Anticipating results of subsequent papers, it should be mentioned that the $3 \mathrm{FD}$ simulations with same set of parameters described here also reproduce other observables, 


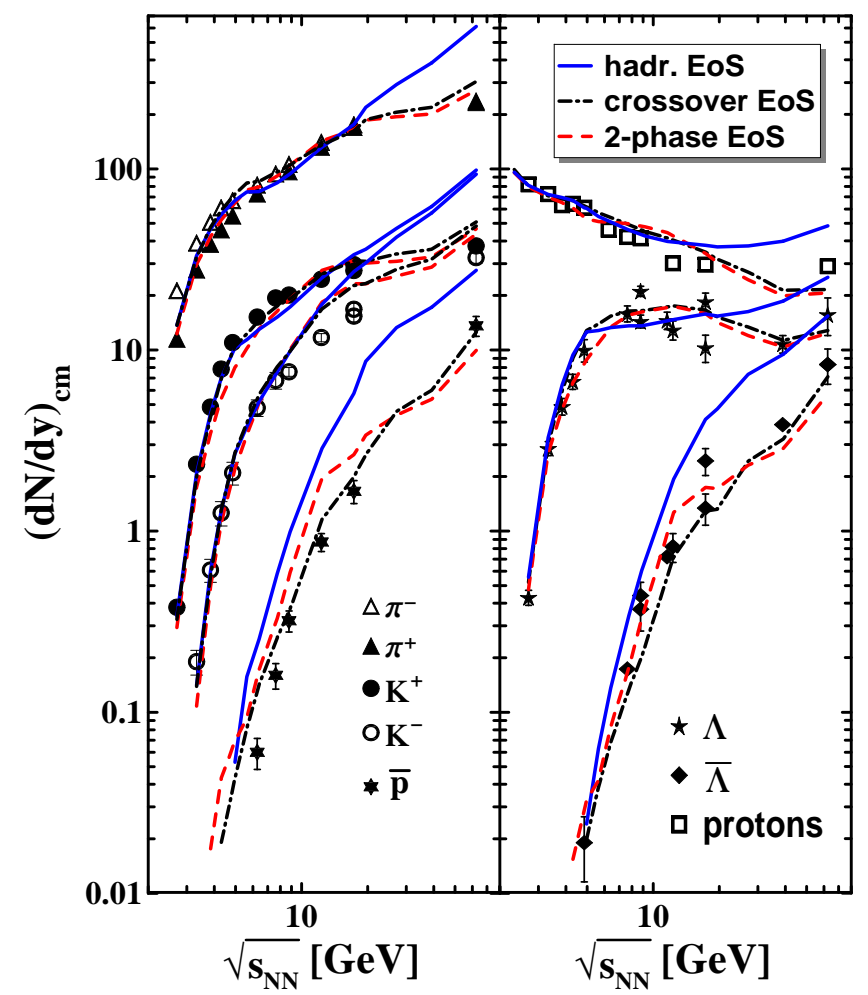

FIG. 9: (Color online) Midrapidity densities of various produced particles as functions of the incident center-of-mass energy of colliding nuclei predicted by 3FD calculations with different EoS's. Experimental data are from compilation of Ref. 50 complemented by recent data from STAR collaboration [58] and latest update of the compilation of NA49 numerical results [59, 60].

of course, with different degree of success depending on applied EoS. As an example, Fig. 9demonstrates excitation functions of midrapidity values of various produced particles. As before, results for top calculated energy of $\sqrt{s_{N N}}=62.4 \mathrm{GeV}$ should be taken with care, since accurate computation is still unavailable for this energy. The strangeness production at low incident energies is overestimated within the 3FD model. This is not sur-

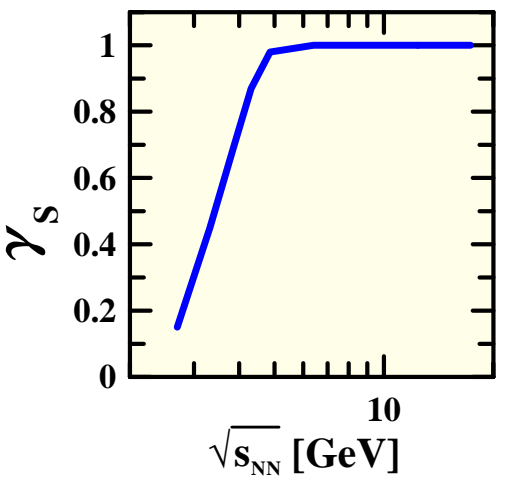

FIG. 10: (Color online) Strangeness suppression factor as a function of the center-of-mass energy of colliding nuclei.

prising, since any EoS in the 3FD model is based on the grand canonical ensemble. This shortcoming can be easily cured by introduction of a phenomenological factor $\gamma_{S}$ 61], which accounts for an additional strangeness suppression due to constraints of canonical ensemble. The midrapidity densities of strange particles, displayed in Fig. 9 are multiplied by $\gamma_{S}$ factor, which in its turn is presented in Fig. 10. As seen, at $E_{\text {lab }}>10 \mathrm{~A} \mathrm{GeV}$ there is no need for additional strangeness suppression.

As seen from Fig. 9] the purely hadronic EoS certainly fails at high energies. A preferable EoS is the crossover one, similarly to that for proton rapidity distributions. This calculations, as well as those of other observables, will be discussed in subsequent papers.

\section{Acknowledgements}

Fruitful discussions with I.N. Mishustin, L.M. Satarov and D.N. Voskresensky are gratefully acknowledged. I am grateful to A.S. Khvorostukhin, V.V. Skokov, and V.D. Toneev for providing me with the tabulated 2-phase and crossover EoS's. The calculations were performed at the computer cluster of GSI (Darmstadt). This work was supported by The Foundation for Internet Development (Moscow) and also partially supported by the Russian Ministry of Science and Education grant NS-215.2012.2.
[1] E. Shuryak, Prog. Part. Nucl. Phys. 62, 48 (2009); ibid.53, 273 (2004) hep-ph/0312227.

[2] G. S. F. Stephans, J. Phys. G 32, S447 (2006) nucl-ex/0607030.

[3] P. Seyboth [NA49 Collaboration], Addedndum-1 to the NA49 Proposal, CERNSPSC- 97-26; M. Gazdzicki, nucl-th/9701050, M. Gazdzicki et al. [NA61/SHINE Collaboration], PoS C POD2006, 016 (2006).

[4] P. Senger, T. Galatyuk, D. Kresan, A. Kiseleva and E. Kryshen, PoS C POD2006 (2006) 018.

[5] A. N. Sissakian, A. S. Sorin and V. D. Toneev, Conf. Proc. C 060726, 421 (2006) nucl-th/0608032.

[6] Yu. B. Ivanov, V. N. Russkikh, and V.D. Toneev, Phys. Rev. C 73, 044904 (2006) nucl-th/0503088.
[7] V. M. Galitsky and I. N. Mishustin, Sov. J. Nucl. Phys. 29, 181 (1979).

[8] Yu. B. Ivanov and V. N. Russkikh, PoS CPOD 07, 008 (2007) arXiv:0710.3708 [nucl-th]].

[9] V. N. Russkikh and Yu. B. Ivanov, Phys. Rev. C 74 (2006) 034904 nucl-th/0606007.

[10] Yu. B. Ivanov and V. N. Russkikh, Eur. Phys. J. A 37, 139 (2008) nucl-th/0607070. Phys. Rev. C 78, 064902 (2008) arXiv:0809.1001 [nucl-th]].

[11] Yu. B. Ivanov, I. N. Mishustin, V. N. Russkikh, and L. M. Satarov, Phys. Rev. C 80, 064904 (2009) arXiv:0907.4140 [nucl-th]].

[12] A. S. Khvorostukhin, V. V. Skokov, K. Redlich, and V. D. Toneev, Eur. Phys. J. C48, 531 (2006) nucl-th/0605069. 
[13] Yu. B. Ivanov, arXiv:1211.2579 [hep-ph], to be published in Phys. Lett. B.

[14] I.N. Mishustin, V.N. Russkikh, and L.M. Satarov, Yad. Fiz. 48, 711 (1988) [Sov. J. Nucl. Phys. 48, 454 (1988)].

[15] V. N. Russkikh, Yu. B. Ivanov, Yu. E. Pokrovsky, and P. A. Henning, Nucl. Phys. A572, 749 (1994).

[16] I.N. Mishustin, V.N. Russkikh, and L.M. Satarov, Yad. Fiz. 54, 429 (1991) [Sov. J. Nucl. Phys. 54, 260 (1991)].

[17] U. Katscher, D.H. Rischke, J.A. Maruhn, W. Greiner, I.N. Mishustin, and L.M. Satarov, Z. Phys. A346, 209 (1993); A. Dumitru, U. Katscher, J.A. Maruhn, H. Stöcker, W. Greiner, and D.H. Rischke, Phys. Rev. C 51, 2166 (1995) hep-ph/9411358; Z. Phys. A353, 187 (1995) hep-ph/9503347.

[18] J. Brachmann, A. Dumitru, J.A. Maruhn, H. Stöcker, W. Greiner, and D.H. Rischke, Nucl. Phys. A619, 391 (1997) nucl-th/9703032; M. Reiter, A. Dumitru, J. Brachmann, J.A. Maruhn, H. Stöcker, and W. Greiner, Nucl. Phys. A643, 99 (1998) nucl-th/9806010; M. Bleicher, M. Reiter, A. Dumitru, J. Brachmann, C. Spieles, S.A. Bass, H. Stöcker, and W. Greiner, Phys. Rev. C 59, R1844 (1999) hep-ph/9811459; J. Brachmann, A. Dumitru, H. Stöcker, and W. Greiner, Eur. Phys. J. A8, 549 (2000) nucl-th/9912014;

[19] S. Bass, et al., Prog. Part. Nucl. Phys. 41, 225 (1998).

[20] W. Cassing and E. L. Bratkovskaya, Phys. Rept. 308, 65 (1999).

[21] Yu.B. Ivanov, Nucl. Phys. A474, 669 (1987).

[22] Yu.B. Ivanov, I.N. Mishustin, and L.M. Satarov, Nucl. Phys. A433, 713 (1985).

[23] L.M. Satarov, Yad. Fiz. 52, 412 (1990) [Sov. J. Nucl. Phys. 52, 264 (1990)].

[24] V.N. Russkikh, Yu.B. Ivanov, Phys. Rev. C 76, 054907 (2007) nucl-th/0611094.

[25] Yu.B. Ivanov, V.N. Russkikh, Yad. Fiz. 72, 12881294 (2009) [Phys. Atom. Nucl. 72, 1238 (2009)] arXiv:0810.2262 [nucl-th]].

[26] A. V. Merdeev, L. M. Satarov and I. N. Mishustin, Phys. Rev. C 84, 014907 (2011) arXiv:1103.3988 [hep-ph]].

[27] I. N. Mishustin, A. V. Merdeev and L. M. Satarov, Phys. Atom. Nucl. 75, 776 (2012) arXiv:1012.4364 [hep-ph]].

[28] H. Petersen, J. Steinheimer, G. Burau, M. Bleicher, and H. Stocker, Phys. Rev. C 78, 044901 (2008) arXiv:0806.1695v3 [nucl-th]]

[29] J. Steinheimer V. Dexheimer, H. Petersen, M. Bleicher, S. Schramm, and H. Stoecker, Phys. Rev. C 81, 044913 (2010) arXiv:0905.3099 [hep-ph]].

[30] Y. Hama, T. Kodama and O. Socolowski, Jr., Braz. J. Phys. 35, 24 (2005) hep-ph/0407264.

[31] C. Nonaka and M. Asakawa, arXiv:1204.4795 [nucl-th].

[32] Bin Zhang, Lie-Wen Chen, Che Ming Ko, J. Phys. G 35, (2008) 065103.

[33] W. Cassing and E. L. Bratkovskaya, Nucl. Phys. A831, 215 (2009) arXiv:0907.5331 [nucl-th]].

[34] Y. Aoki, G. Endrodi, Z. Fodor, S. D. Katz and K. K. Szabo, Nature 443, 675 (2006) hep-lat/0611014.

[35] I. C. Arsene, L.V. Bravina, W. Cassing, Yu.B. Ivanov, A. Larionov, J. Randrup, V.N. Russkikh, V.D. Toneev, G. Zeeb, D. Zschiesche, Phys. Rev. C 75, 034902 (2007) nucl-th/0609042

[36] J. L. Klay et al. [E-0895 Collaboration], Phys. Rev. C 68, 054905 (2003) nucl-ex/0306033.

[37] J. Barrette et al. (E877 Collab.), Phys. Rev. C 62, 024901 (2000).

[38] B. B. Back et al., (E917 Collab.), Phys. Rev. Lett. 86 , 1970 (2001).

[39] J. Stachel, Nucl. Phys. A654, 119c (1999) nucl-ex/9903007.

[40] H. Appelshäuser et al. (NA49 Collab.), Phys. Rev. Lett. 82, 2471 (1999).

[41] T. Anticic et al. (NA49 Collab.), Phys. Rev. C 69, 024902 (2004).

[42] C. Alt et al. (NA49 Collab.), Phys. Rev. C 73, 044910 (2006) nucl-ex/0512033.

[43] C. Blume (NA49 Collab.), J. Phys. G34, S951 (2007) nucl-ex/0701042.

[44] T. Anticic et al. [NA49 Collaboration], Phys. Rev. C 83, 014901 (2011) arXiv:1009.1747 [nucl-ex]].

[45] E. L. Bratkovskaya, M. Bleicher, M. Reiter, S. Soff, H. Stoecker, M. van Leeuwen, S. A. Bass, and W. Cassing, Phys. Rev. C 69, 054907 (2004) nucl-th/0402026.

[46] H. Weber, E. L. Bratkovskaya, W. Cassing, and H. Stöcker, Phys. Rev. C 67, 014904 (2003) nucl-th/0209079.

[47] H. Weber, E. L. Bratkovskaya, and H. Stoecker, Phys. Rev. C 66, 054903 (2002).

[48] A. B. Larionov, O. Buss, K. Gallmeister, and U. Mosel, Phys. Rev. C 76, 044909 (2007) arXiv:0704.1785 [nuclth]].

[49] M. Wagner, A. B. Larionov, and U. Mosel, Phys. Rev. C 71, 034910 (2005) nucl-th/0411010.

[50] A. Andronic, P. Braun-Munzinger and J. Stachel, Nucl. Phys. A 772, 167 (2006) nucl-th/0511071.

[51] C. Alt et al. [NA49 Collaboration], Phys. Rev. C 68, 034903 (2003) nucl-ex/0303001.

[52] B. I. Abelev et al. [STAR Collab.], Phys. Rev. C 79, 034909 (2009) arXiv:0808.2041 [nucl-ex]].

[53] Yu. B. Ivanov, Phys. Lett. B 690, 358 (2010) arXiv:1001.0670 [nucl-th]].

[54] Y. .B. Ivanov, Phys. At. Nucl. 75621 (2012) [1101.2092 [nucl-th]].

[55] Y. Mehtar-Tani and G. Wolschin, Europhys. Lett. 94, 62003 (2011) arXiv:1102.3134 [hep-ph]].

[56] C. M. Hung and E. V. Shuryak, Phys. Rev. Lett. 75, 4003 (1995) hep-ph/9412360.

[57] E. G. Nikonov, A. A. Shanenko and V. D. Toneev, Heavy Ion Phys. 8, 89 (1998) nucl-th/9802018.

[58] X. Zhu [STAR Collaboration], Acta Phys. Polon. Supp. 5, 213 (2012) arXiv:1203.5183 [nucl-ex]].

[59] C. Blume, M. Gazdzicki, B.Lungwitz, M. Mitrovski, P. Seyboth, and H. Strobele, Compilation of NA49 numerical results, https://edms.cern.ch/document/1075059

[60] C. Blume and C. Markert, Prog. Part. Nucl. Phys. 66, 834 (2011) arXiv:1105.2798 [nucl-ex]].

[61] P. Koch, B. Muller and J. Rafelski, Phys. Rept. 142, 167 (1986). 